\title{
Determining the optimal time for liberation from renal replacement therapy in critically ill patients: a systematic review and meta- analysis (DOnE RRT)
}

Riley Jeremy Katulka ${ }^{1}$ D, Abdalrhman Al Saadon', Meghan Sebastianski ${ }^{2}$, Robin Featherstone ${ }^{2,3}$, Ben Vandermeer ${ }^{2,3}$, Samuel A. Silver ${ }^{4}$, R. T. Noel Gibney ${ }^{1}$, Sean M. Bagshaw ${ }^{1}$ and Oleksa G. Rewa ${ }^{1^{*}}$

\begin{abstract}
Introduction: Renal replacement therapy (RRT) is associated with high mortality and costs; however, no clinical guidelines currently provide specific recommendations for clinicians on when and how to stop RRT in recovering patients. Our objective was to systematically review the current evidence for clinical and biochemical parameters that can be used to predict successful discontinuation of RRT.
\end{abstract}

Methods: A systematic review and meta-analysis were performed with a peer-reviewed search strategy combining the themes of renal replacement therapy (IHD, CRRT, SLED), predictors of successful discontinuation or weaning (defined as an extended period of time free from further RRT), and patient outcomes. Major databases were searched and citations were screened using predefined criteria. Studied parameters were reported and, where possible, data was analyzed in the pooled analysis.

Results: Our search yielded 23 studies describing 16 variables for predicting the successful discontinuation of RRT. All studies were observational in nature. None were externally validated. Fourteen studies described conventional biochemical criteria used as surrogates of glomerular filtration rate (serum urea, serum creatinine, creatinine clearance, urine urea excretion, urine creatinine excretion). Thirteen studies described physiologic parameters such as urine output before and after cessation of RRT, and 13 studies reported on newer kidney biomarkers, such as serum cystatin $C$ and serum neutrophil gelatinase-associated lipocalin (NGAL). Six studies reported sensitivity and specificity characteristics of multivariate models. Urine output prior to discontinuation of RRT was the most-studied variable, with nine studies reporting. Pooled analysis found a sensitivity of $66.2 \%$ (95\% Cl, 53.6-76.9\%) and specificity of $73.6 \%$ (95\% Cl, 67.5-79.0\%) for urine output to predict successful RRT discontinuation. Due to heterogeneity in the thresholds of urine output used across the studies, an optimal threshold value could not be determined.

Conclusions: Numerous variables have been described to predict successful discontinuation of RRT; however, available studies are limited by study design, variable heterogeneity, and lack of prospective validation. Urine output prior to discontinuation of RRT was the most commonly described and robust predictor. Further research should focus on the determination and validation of urine output thresholds, and the evaluation of additional clinical and biochemical parameters in multivariate models to enhance predictive accuracy.

Keywords: Systematic review, Renal replacement therapy, Prediction, Acute kidney injury, Intensive care unit, Biomarkers, Creatinine

\footnotetext{
* Correspondence: rewa@ualberta.ca

'Department of Critical Care Medicine, Faculty of Medicine and Dentistry,

University of Alberta, 2-124E Clinical Sciences Building 8440112 St. NW,

Edmonton, Alberta T6G 2B7, Canada

Full list of author information is available at the end of the article
}

(c) The Author(s). 2020 Open Access This article is distributed under the terms of the Creative Commons Attribution 4.0 International License (http://creativecommons.org/licenses/by/4.0/), which permits unrestricted use, distribution, and reproduction in any medium, provided you give appropriate credit to the original author(s) and the source, provide a link to the Creative Commons license, and indicate if changes were made. The Creative Commons Public Domain Dedication waiver (http://creativecommons.org/publicdomain/zero/1.0/) applies to the data made available in this article, unless otherwise stated. 


\section{Introduction}

Acute kidney injury (AKI) is a common problem encountered in the intensive care unit (ICU), estimated to occur in up to $60 \%$ of all critically ill patients, depending on the definition [1]. When AKI progresses in severity, treatment with renal replacement therapy (RRT) may be initiated. RRT is currently applied in $23.5 \%$ of ICU patients with AKI (i.e., 13.5\% of all ICU admitted to the ICU), with utilization growing by over $10 \%$ per year over the past decade $[2,3]$. Recent large randomized clinical trials (RCTs) in critical care nephrology have focused on the optimal timing of initiation of acute RRT [4-6]. However, less is known about the ideal circumstances in which RRT may be successfully discontinued. RRT is a complex and expensive therapy, with complications including catheter-associated infections, hemorrhage, hemodynamic instability, and delayed renal recovery [7-15]. It is thus imperative to recognize when a patient may be safely liberated from this treatment. The Kidney Disease Improving Global Outcomes (KDIGO) organization has stated in their 2012 Clinical Practice Guidelines for Acute Kidney Injury that RRT should be discontinued "when it is no longer required, either because intrinsic kidney function has recovered to the point that it is adequate to meet patient needs, or because RRT is no longer consistent with the goals of care." [16]. However, this recommendation was based on expert opinion and lacks specific guidance for how clinicians should assess patients for suitability to discontinue RRT.

Numerous parameters have been evaluated to help identify patients for whom RRT may be safely discontinued, including traditional biochemical markers of kidney function (creatinine, urea, and estimates of glomerular filtration rate (GFR) [17-26]), clinical findings such as urine output $[21-23,26,27]$, and newer kidney biomarkers including neutrophil gelatinase-associated lipocalin (NGAL) [19, 28, 29] and serum cystatin C [28-31]. Despite many criteria being evaluated in the existing literature, the available evidence has yet to be rigorously synthesized. Our objective was to conduct a systematic review and meta-analysis to identify predictors of successful discontinuation of acute RRT among critically ill patients with AKI.

\section{Methods}

We performed a systematic review using methodological approaches outlined in the Cochrane Handbook for Systematic Reviews of Interventions [32] and described according to the Preferred Reporting Items for Systematic Meta-Analyses (PRISMA) guideline [33]. A PRISMA checklist is available as Additional file 1. Research ethics approval was not required. This systematic review was registered at PROSPERO (2018-0306, CRD42018074615), and the protocol was published separately [34].

\section{Search strategy}

The search strategy was developed in consultation with a research librarian and independently peer-reviewed by a second librarian. We searched electronic databases: Ovid MEDLINE (1946-), Ovid Embase (1988-), and Wiley Cochrane Library (inception-) on October 10, 2017, with an updated search on April 8, 2019. Our search strategy combined concepts related to renal replacement therapy (i.e., intermittent hemodialysis (IHD), slow low-efficiency dialysis (SLED), continuous renal replacement therapy (CRRT)), intensive care (i.e., involving any intensive care unit (ICU) setting), and discontinuation of therapy (i.e., either clinical, physiological, and biochemical parameters of weaning acute RRT) or treatment outcome (Additional file 2). Search results were limited to publications after 1990, when continuous venovenous RRT was initiated. No language limits were applied.

Additional search sources included the trial registry platforms (i.e., ClinicalTrials.gov) and Google Scholar. We also searched meeting abstracts where available using Conference Proceedings Citation Index (Clarivate Analytics) and by hand-searching published proceedings from the following associations and meetings: American Society of Nephrology, Canadian Society of Nephrology, "CRRTonline" (San Diego), European Renal Association - European Dialysis and Transplant Association, European Society of Intensive Care Medicine, International Symposium on Intensive Care and Emergency Medicine (Brussels), National Kidney ?>Foundation, and Society of Critical Care Medicine. Search results were exported and screened in EndNote X7 (Thomson Reuters, Philadelphia, Pennsylvania). See Additional file 2 for the complete search strategy.

\section{Study selection}

Eligible articles were identified through a two-phase process. In the first phase, two authors (AA, RJK) independently reviewed the titles and abstracts of all retrieved articles and documents. Disagreements were resolved through discussion or adjudication by a third author (OGR). In the second phase, full texts of the selected articles were reviewed by the same two authors independently and reviewed for eligibility using standard, predefined criteria. Disagreements were resolved through a discussion with a third author (OGR).

\section{Eligibility criteria}

Studies were included if they mentioned all of the following themes: (1) intensive care (i.e., intended to refer 
to patients supported in an ICU setting capable of providing invasive mechanical ventilation or vasoactive therapy), (2) renal replacement therapy (i.e., IHD, SLED, CRRT), and (3) described parameters associated with weaning or discontinuation (i.e., clinical, physiological, and biochemical parameters). Additionally, we only included adult patients (i.e., age greater or equal to 18 years old) for this review. Studies which did not mention all of these themes were excluded.

\section{Risk of bias assessment}

Study methodological quality was independently rated by two authors (AA, RK) using the Newcastle-Ottawa Scale (NOS) for observational studies [35]. Observational studies were rated as high quality if they had a total score of $6-9$, moderate quality with a score of 4 or 5 , and poor quality if the score was 3 or lower. In order to account for potential bias due to population selection in observational trials, the NOS score for comparability was based on whether included studies accounted for patients with factors that would influence the predictability of RRT discontinuation, such as pre-existing chronic kidney disease (CKD) or prior RRT use in included cohorts. Disagreements were resolved through a discussion with a third author (OGR). The overall quality of evidence and certainty of outcome measures reported was further assessed according to the GRADE framework. A summary of findings' table was prepared using the GRADEpro Guideline Development Tool (https://gradepro.org) for the pooled analysis. Full results of the risk of bias assessment are available in Additional file 3.

\section{Data analysis}

Two-by-two tables of true/false positives/negatives were constructed from exposures and outcomes of weaning parameters reported from studies where available. When these quantities were not explicitly described, they were computed from other available data (e.g., sensitivity/specificity) where possible. When four or more studies reported on the same parameter in a sufficiently homogenous manner, the sensitivity and specificity were simultaneously pooled in a statistical meta-analysis using the bi-variate random effects method [36]. This model assumes that the correlated logit transformed values of the sensitivity and specificity are correlated and follow a bi-variate normal distribution, from which we can estimate not only simultaneous parameter estimates, but also a hierarchical summary receiver operating characteristic (AUROC) curve for the presented data. Review Manager (Version 5.3.5, Copenhagen, Denmark) was used to create the forest plots, while Stata (Version 14.2, College Station, Texas) was used to compute the bivariate estimates.

\section{Results}

\section{Search results}

Our initial search yielded 3031 citations and our updated search yielded an additional 924 citations. Twenty-three articles fulfilled all inclusion criteria (Fig. 1). This consisted of 18 full-text articles and five conference abstracts, representing two case-control studies, 15 retrospective cohort studies, and six prospective cohort studies (Table 1). There were no randomized controlled trials. All studies were published in English.

\section{Study quality}

Study quality was generally rated as high for included observational studies, with a mean NOS score of seven (range 4-9) and no studies being rated as poor quality (see Additional file 3). Twenty studies were rated as high quality and two studies were rated as moderate quality. One conference abstract could not be rated due to insufficient information. Regarding the use of urine output as a predictor of successful RRT discontinuation, the overall certainty of the evidence was rated as very low given methodological limitations inherent to the included retrospective observational studies resulting in the risk of bias, and imprecision in the reported values (Fig. 2). There was consensus among authors as to the quality of the included studies.

\section{Markers of RRT weaning}

A total of 16 variables predictive of RRT weaning and five multivariate models were assessed in 46 instances (Table 1). Weaning variables were grouped into four categories: conventional biochemical criteria (Table 2; $n=$ 14; 29.8\%), kidney biomarkers (Table 3; $n=13 ; 27.7 \%$ ), physiologic criteria (Table $4,5,6 ; n=13 ; 27.7 \%$ ), and multivariate models (Table $7 ; n=5 ; 14.9 \%$ ). Urine output was the most commonly described variable. A total of nine studies described urine output criteria prior to RRT discontinuation and four studies described urine output after RRT discontinuation.

\section{Studies retrieved and parameters identified in a systematic review}

There was a significant heterogeneity across studies in the definitions of "successful" RRT discontinuation and in the thresholds used to define weaning criteria. Most studies defined successful discontinuation as a specified period during which the patient did not receive further RRT; however, the periods specified varied and ranged from 3 days [37] to 60 days [30], with 7 days being the most frequently used (seven studies). Where multiple studies reported on the same variable, the threshold values with optimal predictive accuracy varied, as did the timing of measurement in relation to RRT discontinuation. For cystatin $\mathrm{C}$, two studies measured values 


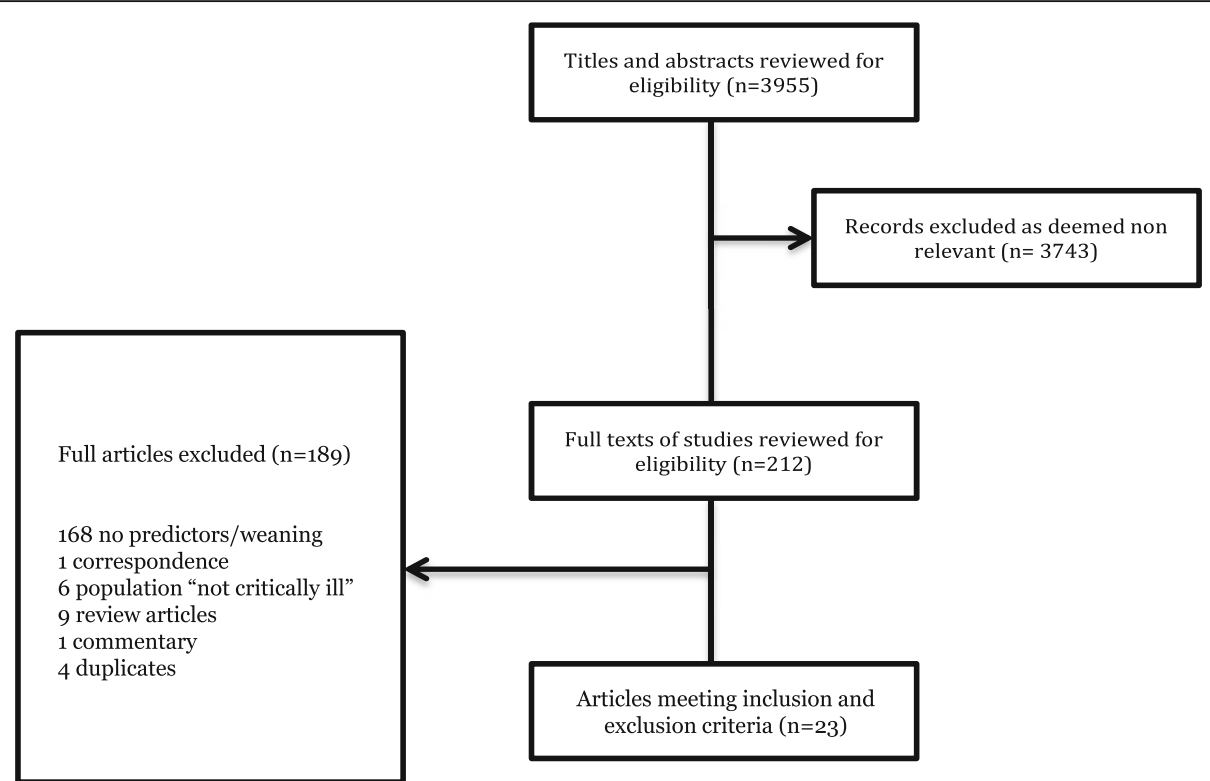

Fig. 1 PRISMA flow diagram of retrieved and included records. Of the 23 included trials, 5 were abstracts and 18 were full text

prior to RRT discontinuation [29, 31], and three measured values following discontinuation [28-30] (Table 3). The optimal threshold values ranged from 1.85 to 2.98 $\mathrm{mg} / \mathrm{L}$, respectively. The predictive accuracy of serum creatinine was assessed in two studies at RRT initiation $[17,18]$, and in four studies at the time of discontinuation $[19,20]$, with threshold levels ranging from 224 to $300 \mu \mathrm{mol} / \mathrm{L}$ (Table 2). Due to variation in the timing of measurement and threshold values, the pooled analysis was not feasible.

\section{Urine output prior to discontinuation of RRT to predict successful weaning}

Urine output was reported in numerous studies, both before and after discontinuation. We felt that the timing of measurement relative to discontinuation (before or after) was the most clinically important characteristic affecting the heterogeneity of reporting; accordingly, we pooled data separately based on the timing of measurement. Urine output prior to RRT discontinuation was sufficiently homogenous across studies to perform pooled analysis (Fig. 2, Table 4). We found a pooled sensitivity of $66.2 \%$ (95\% CI, 53.6 to 76.9 ), specificity of $73.6 \%$ (95\% CI, 67.5 to 79.0 ), $L R+2.91$, and LR -0.43 for urine output (Fig. 2). Estimation of an optimal threshold to discriminate "successful" RRT discrimination was not feasible due to variation across studies, with thresholds ranging from $191 \mathrm{~mL} / 24 \mathrm{~h}$ [37] to 1720 $\mathrm{mL} / 24 \mathrm{~h}$ [17]. Overall certainty of evidence regarding this parameter was graded as very low (Fig. 2).

\section{Urine output after discontinuation of RRT to predict successful weaning}

Urine output following RRT discontinuation was assessed in four studies and had a moderate AUC to predict successful RRT discontinuation (Table 5). Due to the low number of studies and heterogeneity, the pooled analysis was not feasible.

\section{Effect of diuretic use on urine output test characteristics to predict successful discontinuation of RRT}

Diuretic use was associated with successful RRT discontinuation in three studies [21, 37, 42], although test characteristics were not reported. The effect of a diuretic challenge on the predictive accuracy of urine output was assessed in four studies [17, 21, 27, 37] (Table 6). In two small studies [17, 27], there was a suggestion of improved predictive accuracy in patients who had received diuretics. However, a larger study [21] found that urine output was a less reliable predictor of successful discontinuation when diuretics were given and another study did not compare the accuracy of urine output with or without diuretics [37]. Diuretics used include furosemide as an intermittent dose [21, 27, 37, 42] or infusion [27, 37], spironolactone [37], thiazides [37], or other diuretics $[21,42]$. No comparative analysis has been performed on the accuracy of urine output to predict RRT discontinuation among diuretic responders versus non-responders. Because the effect that concomitant diuretic exposure may have on the predictive capacity of urine output for RRT discontinuation remains uncertain, our pooled 
Table 1 Studies retrieved and parameters identified in a systematic review

\begin{tabular}{|c|c|c|c|c|c|c|}
\hline Study & Study type & Population & $\begin{array}{l}\text { \#Patients with } \\
\text { discontinuation } \\
\text { of RRT }\end{array}$ & $\begin{array}{l}\text { Quality } \\
\text { (NOS) }\end{array}$ & Parameters identified & $\begin{array}{l}\text { Definition of weaning } \\
\text { success }\end{array}$ \\
\hline $\begin{array}{l}\text { Chen et al. } \\
\text { [19] }\end{array}$ & $\begin{array}{l}\text { Prospective } \\
\text { cohort }\end{array}$ & Adult patients receiving CRRT & 78 & 9 & $\begin{array}{l}\text { Urine output, plasma NGAL, } \\
\text { serum } \mathrm{Cr} \text { at discontinuation }\end{array}$ & $\begin{array}{l}\text { Survival with no } \\
\text { requirement for RRT within } \\
7 \text { days }\end{array}$ \\
\hline $\begin{array}{l}\text { Yoshida et al. } \\
{[17]}\end{array}$ & $\begin{array}{l}\text { Retrospective } \\
\text { cohort }\end{array}$ & $\begin{array}{l}\text { General ICU patients requiring } \\
\text { RRT }\end{array}$ & 38 & 8 & $\begin{array}{l}\text { Serum } \mathrm{Cr} \text {, urine output, } \\
\text { eGFR, kinetic eGFR at } \\
\text { discontinuation }\end{array}$ & $\begin{array}{l}\text { Survival off CRRT } \times 48 \mathrm{~h} \text {, } \\
\text { off IHD for } 7 \text { days }\end{array}$ \\
\hline Jeon et al. [37] & $\begin{array}{l}\text { Retrospective } \\
\text { cohort }\end{array}$ & Adult patients with AKI on CRRT & 517 & 9 & $\begin{array}{l}\text { Urine output, multivariate } \\
\text { model at discontinuation }\end{array}$ & $\begin{array}{l}\text { Survival with no re- } \\
\text { initiation of RRT within } 3 \\
\text { days }\end{array}$ \\
\hline $\begin{array}{l}\text { Itenov et al. } \\
\text { [38] }\end{array}$ & $\begin{array}{l}\text { Prospective } \\
\text { cohort }\end{array}$ & Adult patients with AKI & 719 & 8 & Multivariate model & $\begin{array}{l}\text { Survival with no re- } \\
\text { initiation of RRT for } 5 \text { days }\end{array}$ \\
\hline Kim et al. [28] & $\begin{array}{l}\text { Prospective } \\
\text { cohort }\end{array}$ & ICU patients weaned from CRRT & 89 & 8 & $\begin{array}{l}\text { Serum cystatin C, plasma } \\
\text { NGAL, urine output }\end{array}$ & $\begin{array}{l}\text { Survival with no re- } \\
\text { initiation of RRT for } 14 \text { days }\end{array}$ \\
\hline $\begin{array}{l}\text { Raurich et al. } \\
{[27]}\end{array}$ & $\begin{array}{l}\text { Retrospective } \\
\text { cohort }\end{array}$ & $\begin{array}{l}\text { ICU patients requiring CRRT who } \\
\text { underwent weaning tests }\end{array}$ & 67 & 9 & $\begin{array}{l}\text { Urine output with/without } \\
\text { diuretics, multivariate } \\
\text { model }\end{array}$ & $\begin{array}{l}\text { Urine output recovered, } \\
\text { RRT not required }\end{array}$ \\
\hline $\begin{array}{l}\text { Romero- } \\
\text { Gonzalez et al. } \\
\text { [39] }\end{array}$ & $\begin{array}{l}\text { Retrospective } \\
\text { cohort }\end{array}$ & $\begin{array}{l}\text { Patients with AKI treated with } \\
\text { CRRT }\end{array}$ & 37 & - & Urine output & $\begin{array}{l}\text { Independence from RRT } 14 \\
\text { days after discontinuation }\end{array}$ \\
\hline $\begin{array}{l}\text { Yang et al. } \\
{[29]}\end{array}$ & $\begin{array}{l}\text { Retrospective } \\
\text { cohort }\end{array}$ & $\begin{array}{l}\text { ICU patients weaned from CRRT, } \\
\text { PIRRT, IHD }\end{array}$ & 302 & 7 & Serum cystatin $C$ & $\begin{array}{l}\text { Survival with no } \\
\text { requirement for RRT } 30 \\
\text { days after discharge }\end{array}$ \\
\hline $\begin{array}{l}\text { Yang et al. } \\
{[30]}\end{array}$ & $\begin{array}{l}\text { Prospective } \\
\text { observational } \\
\text { study }\end{array}$ & $\begin{array}{l}\text { ICU patients who weaned from } \\
\text { CRRT }\end{array}$ & 102 & 7 & Serum cystatin $C$ & $\begin{array}{l}\text { Survival at } 60 \text { days with } \mathrm{Cr} \\
\text { no more than } 1.5 \mathrm{X} \text { baseline }\end{array}$ \\
\hline $\begin{array}{l}\text { Aniort et al. } \\
{[25]}\end{array}$ & $\begin{array}{l}\text { Retrospective } \\
\text { cohort }\end{array}$ & $\begin{array}{l}\text { ICU patients receiving IHD for at } \\
\text { least } 7 \text { days }\end{array}$ & 67 & 8 & $\begin{array}{l}\text { Daily urine urea, eUrea, } \\
\text { urine output }\end{array}$ & $\begin{array}{l}\text { No requirement for further } \\
\text { dialysis sessions during ICU } \\
\text { stay }\end{array}$ \\
\hline $\begin{array}{l}\text { Katayama } \\
\text { et al. [22] }\end{array}$ & $\begin{array}{l}\text { Retrospective } \\
\text { cohort }\end{array}$ & $\begin{array}{l}\text { General ICU patients receiving } \\
\text { CRRT }\end{array}$ & 116 & 8 & Urine output, $\mathrm{Cr}$ & $\begin{array}{l}\text { Survival with no re- } \\
\text { initiation of CRRT for } 7 \text { days }\end{array}$ \\
\hline Han et al. [18] & $\begin{array}{l}\text { Retrospective } \\
\text { cohort }\end{array}$ & $\begin{array}{l}\text { General ICU patients requiring } \\
\text { CRRT }\end{array}$ & 160 & 7 & Multivariate model, $\mathrm{Cr}$ & $\begin{array}{l}\text { Complete or partial } \\
\text { recovery of AKI within } 2 \\
\text { weeks }\end{array}$ \\
\hline Kim et al. [40] & $\begin{array}{l}\text { Prospective } \\
\text { cohort }\end{array}$ & $\begin{array}{l}\text { General ICU patients requiring } \\
\text { RRT }\end{array}$ & 89 & 4 & Cystatin C-based eGFR & $\begin{array}{l}\text { Survival with no re- } \\
\text { initiation of RRT for } 14 \text { days }\end{array}$ \\
\hline $\begin{array}{l}\text { Viallet et al. } \\
{[26]}\end{array}$ & $\begin{array}{l}\text { Retrospective } \\
\text { cohort }\end{array}$ & $\begin{array}{l}\text { Adult patients who received } \\
\text { CRRT, IHD or SLED and survived } \\
\text { ICU stay }\end{array}$ & 26 & 7 & Urine output, urine $\mathrm{Cr}$ & $\begin{array}{l}\text { Cessation of RRT for at least } \\
15 \text { days }\end{array}$ \\
\hline $\begin{array}{l}\text { Gleeson et al. } \\
{[23]}\end{array}$ & $\begin{array}{l}\text { Retrospective } \\
\text { cohort }\end{array}$ & $\begin{array}{l}\text { General ICU patients requiring } \\
\text { RRT }\end{array}$ & 67 & 6 & $\begin{array}{l}\text { Residual creatinine } \\
\text { clearance }\end{array}$ & Not specified \\
\hline $\begin{array}{l}\text { Ohnuma et al. } \\
\text { [41] }\end{array}$ & $\begin{array}{l}\text { Retrospective } \\
\text { cohort }\end{array}$ & $\begin{array}{l}\text { General ICU patients requiring } \\
\text { CRRT or IHD }\end{array}$ & 109 & 5 & Urine output & $\begin{array}{l}\text { Free from RRT for } 7 \text { days } \\
\text { after discontinuation }\end{array}$ \\
\hline $\begin{array}{l}\text { Frohlich et al. } \\
\text { [20] }\end{array}$ & $\begin{array}{l}\text { Retrospective } \\
\text { cohort }\end{array}$ & $\begin{array}{l}\text { General ICU patients requiring } \\
\text { CRRT }\end{array}$ & 53 & 6 & $\begin{array}{l}2 \mathrm{~h} \mathrm{CrCl}, \mathrm{Cr} \text {, urine output at } \\
\text { discontinuation }\end{array}$ & $\begin{array}{l}\text { Free from RRT for } 7 \text { days } \\
\text { after discontinuation }\end{array}$ \\
\hline Heise [42] & $\begin{array}{l}\text { Retrospective } \\
\text { cohort }\end{array}$ & $\begin{array}{l}\text { Surgical ICU patients requiring } \\
\text { CRRT }\end{array}$ & 222 & 9 & Multivariate model & $\begin{array}{l}\text { Discharged from ICU with } \\
\text { no further RRT during } \\
\text { hospital stay }\end{array}$ \\
\hline $\begin{array}{l}\text { Zhang et al. } \\
\text { [31] }\end{array}$ & $\begin{array}{l}\text { Retrospective } \\
\text { cohort }\end{array}$ & $\begin{array}{l}\text { General ICU patients requiring } \\
\text { CRRT }\end{array}$ & 145 & 8 & Serum cystatin $C$ & $\begin{array}{l}\text { Survivors who were not } \\
\text { dependent on RRT }\end{array}$ \\
\hline Solymos [43] & $\begin{array}{l}\text { Retrospective } \\
\text { cohort }\end{array}$ & $\begin{array}{l}\text { General ICU patients requiring } \\
\text { CRRT }\end{array}$ & 23 & 6 & $2 \mathrm{~h} \mathrm{CrCl}$ & $\begin{array}{l}\text { Free from RRT for } 5 \text { days } \\
\text { after discontinuation }\end{array}$ \\
\hline $\begin{array}{l}\text { Franzen et al. } \\
{[44]}\end{array}$ & $\begin{array}{l}\text { Retrospective } \\
\text { cohort }\end{array}$ & $\begin{array}{l}\text { Medical ICU patients requiring } \\
I H D\end{array}$ & 20 & 7 & IHD ultrafiltration & No need for long-term RRT \\
\hline $\begin{array}{l}\text { Uchino et al. } \\
\text { [21] }\end{array}$ & $\begin{array}{l}\text { Prospective } \\
\text { cohort }\end{array}$ & $\begin{array}{l}\text { General ICU patients requiring } \\
\text { CRRT }\end{array}$ & 313 & 7 & Urine output, $\mathrm{Cr}$ & $\begin{array}{l}\text { Free from RRT for } 7 \text { days } \\
\text { after discontinuation }\end{array}$ \\
\hline
\end{tabular}


Table 1 Studies retrieved and parameters identified in a systematic review (Continued)

\begin{tabular}{|c|c|c|c|c|c|c|}
\hline Study & Study type & Population & $\begin{array}{l}\text { \#Patients with } \\
\text { discontinuation } \\
\text { of RRT }\end{array}$ & $\begin{array}{l}\text { Quality } \\
\text { (NOS) }\end{array}$ & Parameters identified & $\begin{array}{l}\text { Definition of weaning } \\
\text { success }\end{array}$ \\
\hline Wu et al. [45] & Case control & $\begin{array}{l}\text { Surgical ICU patients requiring } \\
\text { CRRT or IHD }\end{array}$ & 64 & 7 & Multivariate model & $\begin{array}{l}\text { Free from RRT for } 30 \text { days } \\
\text { after discontinuation }\end{array}$ \\
\hline
\end{tabular}

analysis does not adjust for diuretic-induced urine output (Fig. 3).

\section{Multivariate models used to predict successful discontinuation of RRT}

Several multivariate models were studied and those which described the operative characteristics of variables, such as sensitivity, specificity, and AUROC are included in Table 7. The timing of these models varied, with one model integrating NT-proBNP, APACHE II score, urine output, and serum creatinine at RRT initiation (estimated AUROC of 0.70 [18]), and another model integrating age, sex, urine output, and serum creatinine during the first $24 \mathrm{~h}$ of admission (estimated AUROC of 0.73 [38]). The remaining three models included variables either at RRT discontinuation [17, 45] or at approximately $8 \mathrm{~h}$ after RRT discontinuation [42]. These models had good to excellent discrimination. In the study by Yoshida et al. [17], the model included urine output at day 0 (the day of CRRT discontinuation) and kinetic eGFR at day 1 (the day after CRRT discontinuation) and the AUROC was 0.93 for prediction of successful RRT discontinuation. None of the studies that included multivariable models have been externally validated.

\section{Secondary outcomes}

Analysis of the relationships between reported weaning parameters and several secondary outcomes (new CKD, RRT duration, RRT use post-ICU discharge) was planned, but not carried out, as the available data made such analysis unfeasible (Additional file 4). New CKD and RRT use post-discharge were infrequently reported in included studies, and when reported were variably defined [27, 38, 39]. While RRT duration was specified in most studies, it was only analyzed in relation to weaning parameters in four instances [21, 22, 42, 45]. In three of these studies, RRT duration was included as a component of multivariate models used to predict successful discontinuation, as opposed to being the predicted endpoint. Given the infrequent reporting, heterogeneous definitions, and confounding effect of being both

Question: Should urine output prior to discontinuation of renal replacement therapy (RRT) be used to diagnose successful discontinuation in patients undergoing acute RRT?

\begin{tabular}{|l|l|}
\hline Sensitivity & $0.67(95 \% \mathrm{Cl}: 0.48$ to 0.81$)$ \\
\hline Specificity & $0.77(95 \% \mathrm{Cl}: 0.71$ to 0.83$)$ \\
\hline
\end{tabular}

\begin{tabular}{|c|c|c|c|c|c|c|c|c|}
\hline \multirow{2}{*}{ Outcome } & \multirow{2}{*}{$\begin{array}{c}\text { № of studies (№ of } \\
\text { patients) }\end{array}$} & \multirow{2}{*}{ Study design } & \multicolumn{5}{|c|}{ Factors that may decrease certainty of evidence } & \multirow{2}{*}{ Test accuracy CoE } \\
\hline & & & Risk of bias ${ }^{1}$ & Indirectness & Inconsistency & Imprecision $^{2}$ & Publication bias & \\
\hline $\begin{array}{l}\text { True positives } \\
\text { (patients with } \\
\text { successful } \\
\text { discontinuation) }\end{array}$ & \multirow[t]{2}{*}{$\begin{array}{l}6 \text { studies } \\
1072 \text { patients }\end{array}$} & \multirow[t]{2}{*}{$\begin{array}{l}\text { Cohort \& case- } \\
\text { control type } \\
\text { studies }\end{array}$} & \multirow[t]{2}{*}{ Very serious } & \multirow[t]{2}{*}{ Not serious } & \multirow[t]{2}{*}{ Not serious } & \multirow[t]{2}{*}{ Serious } & \multirow{2}{*}{$\begin{array}{l}\text { Publication bias } \\
\text { strongly suspected } \\
\text { all plausible } \\
\text { residual } \\
\text { Confounding would } \\
\text { reduce the } \\
\text { demonstrated } \\
\text { effect } \\
\text { No definitive dose } \\
\text { response gradient }\end{array}$} & \multirow[t]{2}{*}{$\begin{array}{l}\oplus \bigcirc 0 \bigcirc \\
\text { VERY LOW }\end{array}$} \\
\hline $\begin{array}{l}\text { False negatives } \\
\text { (patients } \\
\text { incorrectly } \\
\text { classified as not } \\
\text { having successful } \\
\text { discontinuation) }\end{array}$ & & & & & & & & \\
\hline $\begin{array}{l}\text { True negatives } \\
\text { (patients without } \\
\text { successful } \\
\text { discontinuation) }\end{array}$ & \multirow[t]{2}{*}{$\begin{array}{l}6 \text { studies } \\
993 \text { patients }\end{array}$} & \multirow[t]{2}{*}{$\begin{array}{l}\text { Cohort \& case- } \\
\text { control type } \\
\text { studies }\end{array}$} & \multirow[t]{2}{*}{ Very serious } & \multirow[t]{2}{*}{ Not serious } & \multirow[t]{2}{*}{ Not serious } & \multirow[t]{2}{*}{ Serious } & \multirow{2}{*}{$\begin{array}{l}\text { Publication bias } \\
\text { strongly suspected } \\
\text { all plausible } \\
\text { residual } \\
\text { Confounding would } \\
\text { reduce the } \\
\text { demonstrated } \\
\text { effect } \\
\text { No definitive dose } \\
\text { response gradient }\end{array}$} & \multirow[t]{2}{*}{$\begin{array}{l}\oplus 000 \\
\text { VERY LOW }\end{array}$} \\
\hline $\begin{array}{l}\text { False positives } \\
\text { (patients } \\
\text { incorrectly } \\
\text { classified as } \\
\text { having successful } \\
\text { discontinuation) }\end{array}$ & & & & & & & & \\
\hline
\end{tabular}

1. Risk of bias was felt to be very serious due to observational nature of included studies lack of blinding and inconsistent protocol for re-initiating RRT

2. Imprecision was felt to be serious due to the wide range of reported values and confidence intervals.

Fig. 2 Summary of findings' table for urine output prior to discontinuation of RRT 
Table 2 Conventional biochemical criteria used to predict successful discontinuation of RRT

\begin{tabular}{|c|c|c|c|c|c|c|c|c|}
\hline Test/parameter & Value/cut-off & Timing & RRT modality & Sn & $\mathrm{Sp}$ & OR & AUROC & Publication \\
\hline \multicolumn{9}{|l|}{ Serum creatinine } \\
\hline & $299.68 \mathrm{umol} / \mathrm{L}$ & Initiation & CRRT & 0.79 & 0.79 & & 0.75 & Yoshida et al. [17] \\
\hline & Not specified & Initiation & CRRT & & & & 0.59 & Han et al. [18] \\
\hline & $224 \mathrm{umol} / \mathrm{L}$ & Discontinuation & CRRT & 0.72 & 0.77 & & 0.76 & Chen et al. [19] \\
\hline & Per umol/L increase & Discontinuation & CRRT & & & & 0.48 & Frohlich et al. [20] \\
\hline & Not specified & Discontinuation & CRRT & & & & 0.64 & Uchino et al. [21] \\
\hline & Not specified & Discontinuation & CRRT & & & & 0.73 & Katayama et al. [22] \\
\hline \multicolumn{9}{|c|}{ 2-h creatinine clearance } \\
\hline & $23 \mathrm{~mL} / \mathrm{min}$ & $12 \mathrm{~h}$ pre-stop & Not specified & & & 1.11 & 0.82 & Frohlich et al. [20] \\
\hline \multicolumn{9}{|c|}{ Residual creatinine clearance } \\
\hline & Not specified & 24-48 h pre-stop & Not specified & & & & 0.90 & Gleeson et al. [23] \\
\hline \multicolumn{9}{|l|}{ Kinetic eGFR } \\
\hline & $20.58 \mathrm{~mL} / \mathrm{min} / 1.73 \mathrm{~m}^{2}$ & Discontinuation & CRRT & 0.71 & 0.92 & & 0.87 & Yoshida et al. [17] \\
\hline \multicolumn{9}{|l|}{ eGFR } \\
\hline & $26.21 \mathrm{~mL} / \mathrm{min} / 1.73 \mathrm{~m}^{2}$ & D1 post-stop & CRRT & 0.71 & 0.85 & & 0.83 & Yoshida et al. [17] \\
\hline \multicolumn{9}{|c|}{$24 \mathrm{~h}$ urine creatinine } \\
\hline & $>5.2 \mathrm{~mol} / 24 \mathrm{~h}$ & D0 post-stop & CRRT, SLED, & 0.57 & 0.96 & & 0.76 & Viallet et al. [26] \\
\hline & & D1 & $\mathrm{IHD}$ & 0.75 & 0.88 & & 0.86 & \\
\hline & & D2 & & 0.86 & 0.81 & & 0.86 & \\
\hline \multicolumn{9}{|l|}{ Urine urea } \\
\hline & $>148 \mathrm{mmol} / \mathrm{L}$ & Discontinuation & IHD, CRRT & 0.65 & 0.90 & & 0.82 & Aniort et al. [25] \\
\hline \multicolumn{9}{|c|}{ Daily urinary urea excretion } \\
\hline & $>1.35$ mmol/kg/day & Discontinuation & IHD, CRRT & 0.89 & 0.97 & & 0.96 & Aniort et al. [25] \\
\hline
\end{tabular}

predictor and endpoint, we felt that meaningful inferences regarding these parameters could not be derived from the included studies and chose to omit the planned secondary analysis.

\section{Discussion}

Key findings

We found 16 unique parameters that have been evaluated for their ability to predict successful discontinuation of RRT and classified our findings into four categories: physiologic findings (i.e., urine output), conventional biochemical markers of kidney function (creatinine, urea, and estimates of GFR), kidney biomarkers (cystatin C and NGAL), and multivariate models integrating a variety of clinical and biochemical data.

Of all the variables described, urine output was the most commonly studied, with pooled sensitivities and specificities suggesting a modest $(66.2 \%$ [95\% CI, 53.6 to 76.9] and 73.6\% [95\% CI, 67.5 to 79.0]) predictive ability for successful RRT discontinuation for urine output. We found that there was substantial heterogeneity across studies in optimal thresholds for urine output, ranging from $191 \mathrm{~mL} /$ day [37] to over $1700 \mathrm{~mL} /$ day [17].
The effect of a diuretic challenge was variable among included studies, with some studies describing a decrease in the predictive ability of urine volume after diuretic administration [17, 27] and others describing superior discrimination following diuretic administration [21, 37]. The association of diuretic use with successful discontinuation of RRT [21, 37, 42] suggests that augmented diuresis may be an important management strategy to mitigate the need for re-initiation of RRT due to fluid accumulation following initial RRT discontinuation. A randomized controlled trial has previously demonstrated enhanced urinary volume and sodium excretion with infusion of furosemide in patients with resolving ARF; however, no overall improvement in renal outcome was seen, possibly due to infusion of fluids equal to the volume of diuresis in this trial which would have mitigated the potentially beneficial effects of a negative fluid balance [46].

At present, there is currently insufficient data to recommend a specific approach or identify a specific urine output threshold that may reliably predict successful RRT discontinuation. This is due to the fact that urine output was evaluated at different time points (i.e., 
Table 3 Kidney biomarkers used to predict successful discontinuation of RRT

\begin{tabular}{|c|c|c|c|c|c|c|c|c|}
\hline Test/parameter & Value/cut-off & Timing & RRT modality & $\mathrm{Sn}$ & $\mathrm{Sp}$ & OR & AUROC & Publication \\
\hline \multicolumn{9}{|l|}{ Serum cystatin C } \\
\hline & 2.47 mg/L & Initiation & CRRT & 0.95 & 0.54 & & 0.75 & Yang et al. [30] \\
\hline & $2.98 \mathrm{mg} / \mathrm{L}$ & ICU admission & CRRT & 0.81 & 0.84 & 4.76 & 0.87 & Zhang et al. [31] \\
\hline & $2.97 \mathrm{mg} / \mathrm{L}$ & Discontinuation & CRRT & 0.80 & 0.58 & & 0.71 & Yang et al. [30] \\
\hline & $1.85 \mathrm{mg} / \mathrm{L}$ & Discontinuation & CRRT & 0.76 & 0.63 & 0.29 & 0.74 & Kim et al. [28] \\
\hline & Not specified & Discontinuation & CRRT & & & & 0.74 & Yang et al. [29] \\
\hline \multicolumn{9}{|c|}{ Cystatin C-based eGFR } \\
\hline & $32.9 \mathrm{~mL} / \mathrm{min} / 1.73 \mathrm{~m}^{2}$ & Discontinuation & CRRT & 0.65 & 0.76 & 1.25 & 0.75 & Kim et al. [40] \\
\hline \multicolumn{9}{|l|}{ NT-proBNP } \\
\hline & $>15,767$ & Initiation & CRRT & & & 0.54 & 0.58 & Han et al. [18] \\
\hline \multicolumn{9}{|l|}{ NGAL } \\
\hline & Not specified & Discontinuation & CRRT & 0.91 & 0.45 & & 0.65 & Kim et al. [28] \\
\hline & $403 \mathrm{ng} / \mathrm{mL}$ & Discontinuation & CRRT & 0.91 & 0.61 & & 0.81 & Chen et al. [19] \\
\hline & Not specified & Discontinuation & CRRT & & & & 0.66 & Yang et al. [30] \\
\hline \multicolumn{9}{|l|}{ IL-18 } \\
\hline & Not specified & Discontinuation & CRRT & & & & 0.60 & Yang et al. [30] \\
\hline \multicolumn{9}{|l|}{ IL-6 } \\
\hline & Not specified & Discontinuation & CRRT & & & & 0.55 & Yang et al. [30] \\
\hline \multicolumn{9}{|c|}{ Serum osteopontin } \\
\hline & Not specified & Discontinuation & CRRT & & & & 0.61 & Yang et al. [30] \\
\hline
\end{tabular}

preceding and following the exact timing of RRT discontinuation), with or without the actions of diuretics, and with cut-offs that varied greatly between studies. The pooled sensitivity and specificity represent the predictive ability of urine output in general, but the inability to determine an optimal threshold value based on available data currently limits the clinical utility of this parameter.

Kidney biomarkers have shown promise for prognostication in the setting of AKI and have been assessed in several studies evaluating RRT discontinuation and kidney recovery. Cystatin $\mathrm{C}$ was the most commonly studied, and though it appears to have a promising discrimination, due to substantial clinical heterogeneity in the timing of measurement and threshold values used in retrieved studies [28-30], data could not be pooled and definitive inferences on the accuracy of cystatin $\mathrm{C}$ measurement to predict successful RRT discontinuation could not be provided.

Numerous multivariate models have been proposed and generally shown good predictive ability (Table 7).

Table 4 Urine output after discontinuation of RRT to predict successful weaning

\begin{tabular}{|c|c|c|c|c|c|}
\hline Study & Cut-off value & \# Patients & Sensitivity & Specificity & AUROC \\
\hline Aniort et al. [25] & $>8.6 \mathrm{~mL} / \mathrm{kg} / 24 \mathrm{~h}$ & 67 & 0.89 & 0.73 & 0.86 \\
\hline Gleeson et al. [23] & Not specified & 157 & Not estimable & Not estimable & 0.87 \\
\hline Katayama et al. [22] & $100 \mathrm{~mL} /$ day increase & 213 & Not estimable & Not estimable & 0.81 \\
\hline Kim et al. [28] & $>1.26 \mathrm{~mL} / \mathrm{kg} / \mathrm{h}$ & 110 & 0.60 & 0.67 & 0.67 \\
\hline Uchino et al. [21] & $>400$ mL/day (no diuretics) & 1006 & 0.46 & 0.81 & 0.85 \\
\hline Yoshida et al. [17] & $>1720 \mathrm{~mL} / 24 \mathrm{~h}$ & 52 & 0.68 & 0.86 & 0.78 \\
\hline Chen et al. [19] & $>715 \mathrm{~mL} / 24 \mathrm{~h}$ & 110 & 0.83 & 0.87 & 0.85 \\
\hline Jeon et al. [37] & $>191 \mathrm{~mL} / 24 \mathrm{~h}$ & 557 & 0.81 & 0.72 & 0.82 \\
\hline Romero-Gonzalez et al. [39] & $>720 \mathrm{~mL} / 24 \mathrm{~h}$ & 77 & Not estimable & Not estimable & 0.80 \\
\hline \multirow[t]{2}{*}{ POOLED } & & & $0.66(0.54,0.77)$ & $\begin{array}{l}0.77(0.71,0.83) \\
0.74(0.68,0.79)\end{array}$ & \\
\hline & & & $\operatorname{LR}(-) 0.43$ & $\operatorname{LR}(+) 2.91$ & \\
\hline
\end{tabular}


Table $\mathbf{5}$ Urine output prior to discontinuation of RRT to predict successful weaning

\begin{tabular}{|c|c|c|c|c|c|}
\hline Study & Cut-off value & \# Patients & Sensitivity & Specificity & AUROC \\
\hline Raurich et al. [27] & $>178 \mathrm{~mL}$ in the $6 \mathrm{~h}$ post-discontinuation & 86 & 0.90 & 0.89 & 0.91 \\
\hline Viallet [26] & $>2575 \mathrm{~mL} / 24 \mathrm{~h}$ post-discontinuation & 54 & 0.38 & 0.93 & 0.65 \\
\hline Yoshida et al. [17] & > $1709 \mathrm{~mL} / 24 \mathrm{~h}$ post-discontinuation & 52 & 0.76 & 0.79 & 0.77 \\
\hline Han et al. [18] & Not specified & 160 & Not estimable & Not estimable & 0.63 \\
\hline
\end{tabular}

Common features include measures of illness acuity (i.e., SOFA score or APACHE II score), urine output, and variables related to RRT duration (i.e., total days on RRT; number of RRT cycles). The timing of measurement of various parameters comprising a model is important. Among those models whose measurements are taken at the time of ICU admission or RRT initiation $[18,38]$, there is inherent confounding by a competing risk of death as a reason for RRT discontinuation, and there may be lower reliability to predict RRT in the intermediate term. Ideally, such models that integrate a spectrum of clinical information would have the capacity to inform and guide clinical decision-making on when to discontinue RRT. Those models that integrate variables taken at the time of or near the time of RRT discontinuation, along with other important time-varying variables (e.g., changes in acuity and non-kidney organ dysfunction) and which provide a standardized timeframe to assess re-initiation (with standardized criteria for reinitiation) are likely ideally suited to inform practice. Among the most promising models was the one described in the study by Yoshida et al., which combined the urine output on the day of discontinuation of RRT with the kinetic eGFR on the first-day postdiscontinuation (AUROC 0.93) [17]. Importantly, these models would ideally undergo external validation to further assess performance and generalizability. Finally, each of these models utilized only 2 to 4 variables, never exceeding 10 events per variable in the multivariate models, thus minimizing the risk of model overfitting.

\section{Context with prior literature}

Observational studies have shown that early re-initiation of RRT after a failed weaning attempt is associated with greater mortality, although it is unclear if this was more a surrogate of increased or evolving illness severity rather than non-recovery of kidney function and RRT independence [21]. A failed attempt to discontinue RRT may contribute to worsened or exacerbated physiologic profiles, uncertainty in drug dosing, and potentially reexposing patients to the harmful sequelae of AKI, such as fluid accumulation, metabolic acidosis, and retention of metabolic waste.

Unlike weaning from mechanical ventilation, for which there are rigorously evaluated and published protocols $[47,48]$, the paucity of controlled trials to guide discontinuation of RRT has resulted in wide variations in practice $[1,49,50]$, which may contribute to suboptimal care [51]. This may contribute to delays or premature discontinuation of RRT, which can have both patientspecific and health system-specific outcomes and resource implications.

Previous narrative reviews have suggested a weaning attempt of RRT in stable patients when the spontaneous urine output was $>400 \mathrm{~mL} / 24 \mathrm{~h}$ and measured creatinine clearance was $15-20 \mathrm{~mL} / \mathrm{min}$ [52], or when the urine output was $>30 \mathrm{~mL} / \mathrm{h}$ and the 24-h urinary creatinine excretion was $>5.2 \mathrm{mmol} / \mathrm{L}$ [53]. Our study reaffirms the importance of urine output as a clinical marker to help guide RRT discontinuation; however, there is little evidence to determine an optimal threshold urine output value that can be reliably used by clinicians.

Table 6 Effect of diuretic use on urine output test characteristics to predict successful discontinuation of RRT

\begin{tabular}{|c|c|c|c|c|c|}
\hline Study & Cutoff Value & \# Patients & Sensitivity (95\% Cl) & Specificity $(95 \%$ Cl) & AUROC $(95 \% \mathrm{Cl})$ \\
\hline Jeon et al., diuretics [37] & $191 \mathrm{~mL} /$ day & 557 & $81.2(77.6,84.5)$ & $71.6(68.0,75.0)$ & $0.821(0.797,0.845)$ \\
\hline Jeon et al., diuretics (oliguric) [37] & $125 \mathrm{~mL} /$ day & 619 & $72.1(64.6,78.8)$ & $68.8(61.3,75.7)$ & $0.745(0.692,0.798)$ \\
\hline Raurich et al., no diuretics [27] & $178 \mathrm{~mL} / 6 \mathrm{~h}$ & 42 & - & - & $\begin{array}{l}0.73(0.58,0.89) \text { before, } \\
0.85(0.72,0.99) \text { after cessation }\end{array}$ \\
\hline Raurich et al., diuretics [27] & $178 \mathrm{~mL} / 6 \mathrm{~h}$ & 59 & - & - & $\begin{array}{l}0.86(0.76,0.88) \text { before, } \\
0.94(0.88,1.0) \text { after cessation }\end{array}$ \\
\hline Uchino et al., no diuretics [21] & $436 \mathrm{~mL} /$ day & 335 & 46.5 & 80.9 & $0.845(0.799,0.883)$ \\
\hline Uchino et al., diuretics [21] & 2330 mL/day & 194 & - & - & $0.671(0.585,0.750)$ \\
\hline Yoshida et al., no diuretics [17] & 1810 mL/day & 22 & 61.5 & 77.8 & $0.71(0.46,0.88)$ \\
\hline Yoshida et al., diuretics [17] & 1720 mL/day & 30 & 72.0 & 100.0 & $0.84(0.64,0.94)$ \\
\hline
\end{tabular}


Table 7 Multivariate models used to predict successful discontinuation of RRT

\begin{tabular}{|c|c|c|c|c|c|c|c|c|c|}
\hline Test/parameter & Value/cut-off & Timing & \#Patients & Modality & Sn & Sp & OR & AUROC & Publication \\
\hline \multicolumn{10}{|l|}{ Multivariate } \\
\hline & NT-proBNP, APACHE2, UO, Cr & At initiation & 160 & CRRT & & & & 0.70 & Han et al. [18] \\
\hline & Age, gender, UO, Cr & First $24 \mathrm{~h}$ of admission & 719 & CRRT & & & & 0.73 & Itenov et al. [38] \\
\hline & RRT duration, SOFA, oliguria, age & Discontinuation & 64 & $\mathrm{CWH} / \mathrm{IHD}$ & & & & 0.88 & Wu et al. [45] \\
\hline & Urine output, SOFA, \#CRRT cycles & 8 h post-stop & 222 & CRRT & 0.74 & 0.74 & & 0.80 & Heise et al. [42] \\
\hline & Urine output D0, kinetic eGFR D1 & Discontinuation & 38 & CRRT & 0.84 & 1.00 & & 0.93 & Yoshida et al. [17 \\
\hline
\end{tabular}

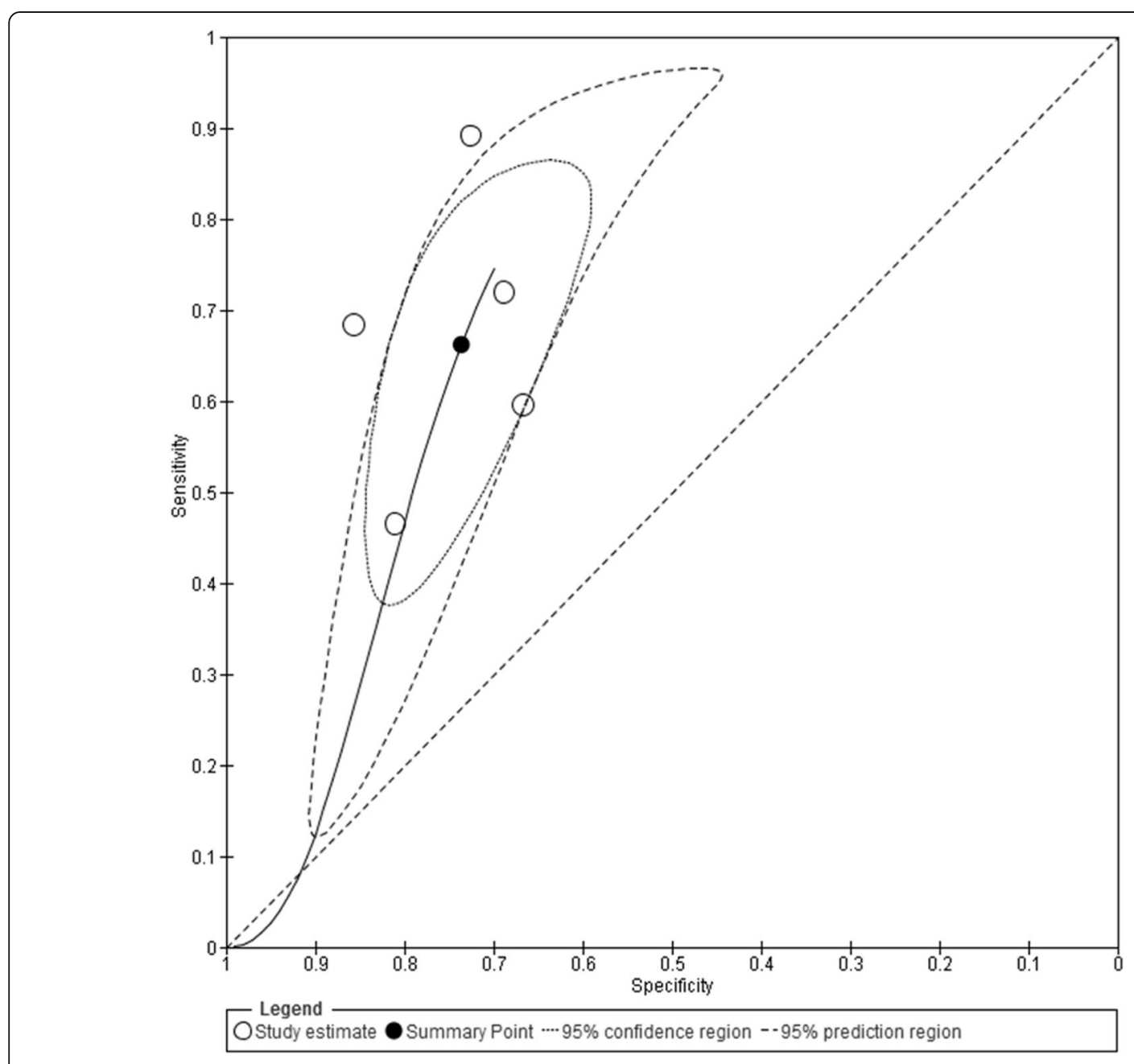

Study  FP FN TNN TN Sens $0.89[0.75,0.97]$
$0.72[0.67,0.76]$
$0.60[0.49,0.70]$
$0.46[0.41,0.52]$
$0.68[0.51,0.82]$ Specificity $(95 \% \mathrm{Cl}) \quad$ Sensitivity $(95 \% \mathrm{Cl})$ Specificity $(95 \% \mathrm{Cl})$ Aniort 2016 Jeon 2018 Kim 2018 $\begin{array}{llll}33 & 14 & 4 & 3\end{array}$ $\begin{array}{llll}279 & 72 & 109 & 159\end{array}$ $0.73[0.58,0.84]$ $\begin{array}{llll}53 & 7 & 36 & 14\end{array}$ $\begin{array}{llll}146 & 41 & 168 & 175\end{array}$ $0.69[0.62,0.75]$ $0.67[0.43,0.85]$ $0.81[0.75,0.86]$ Uchino 2009 $\begin{array}{llll}26 & 2 & 12 & 12\end{array}$

$0.86[0.57,0.98]$

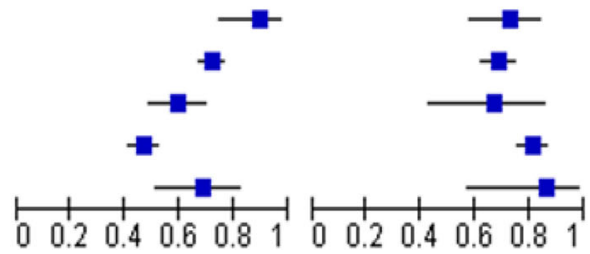

Fig. 3 Pooled analysis for studies using urine output prior to discontinuation of RRT to predict successful weaning 


\section{Implications for future research}

Our study highlights important avenues of future research and reveals significant knowledge gaps in the existing literature. Several markers that have been evaluated appear to have reasonable discrimination, such as daily urinary urea excretion (AUROC of 0.96) [25], and kinetic eGFR (a method developed to reflect true GFR in situations where the serum creatinine is changing dynamically; AUROC of 0.87 in two different studies [17, 24]). However, these have generally be evaluated in small, isolated, retrospective studies and there is a need for replication in larger prospective cohorts before reliable inferences can be made. In addition, further work is needed to understand optimal urine output thresholds, the ideal measurement intervals to predict a successful RRT discontinuation, and further validation across clinical care settings and case-mix.

The predictive ability of urine output could be further augmented to aid clinical decision-making by a combination of patient-specific variables (i.e., age, CKD status) with time-varying and dynamic variables (i.e., daily SOFA score, kinetic GFR, RRT duration) [17, 45]. This would be an important advance in updating clinical practice guidelines with recommendations that are more detailed and clinically prescriptive for clinicians.

\section{Strengths and limitations}

Our review identifies and synthesizes a wide array of physiologic and biochemical markers of weaning success for acute renal replacement therapy. The strengths of our study include the peer-reviewed comprehensive search strategy and a rigorous methodology as outlined in the PRISMA guideline.

Despite these strengths, there are some important limitations in the studies retrieved that warrant consideration. First, although the overall quality of included studies was felt to be high, all data were derived from observational studies, as no randomized trials assessing liberation from RRT were found. Second, many different markers have been studied; however, most have only been evaluated in a single study, have not been replicated, and have not been externally validated. Where markers have been assessed in multiple studies, we found significant heterogeneity in the thresholds used to define positive or negative results, the timing of measurement with respect to the discontinuation of RRT, and in the operational definition used for weaning success. This limited the ability to generalize results and provide a pooled estimate of predictive ability for markers that have been studied in multiple cohorts.

Finally, we were unable to carry out a planned analysis of secondary endpoints such as new CKD, RRT duration, and RRT use post-ICU discharge. With the exception of RRT duration, these endpoints were infrequently reported and none of the included studies related these endpoints to parameters used to predict RRT discontinuation in a way that could be meaningfully aggregated. Subgroup analyses were also planned [34], stratified by age, RRT modality, and CKD status; however, we found that these subgroups were not well described and were heterogeneously defined across studies and thus did not permit further subgroup analysis.

\section{Conclusions}

Our systematic review identified 16 variables for the prediction of successful RRT discontinuation. Where multiple studies reported on the same parameter, the timing of measurement and threshold values used were heterogeneous, making pooled analysis not feasible for most. Urine output prior to discontinuation of RRT was the most-studied variable to predict RRT discontinuation (pooled sensitivity and specificity of $66.2 \%$ and $73.6 \%$ ); however, an optimal threshold value was not determined due also to heterogeneity across retrieved studies. Future work should focus on refinement of a urinary output threshold value and the development and validation of a clinical prediction tool, incorporating urine output with other static and dynamic clinical variables, to better guide clinicians on when to discontinue RRT in ICU settings.

\section{Supplementary information}

Supplementary information accompanies this paper at https://doi.org/10. 1186/s13054-020-2751-8.

Additional file 1. PRISMA Checklist. PRISMA Checklist for DOnE_RRT.

Additional file 2. Search Strategy. Complete search strategy for DOnE_RRT.

Additional file 3. Risk of Bias Assessment. Complete numerical breakdown of.

Additional file 4. Secondary Outcomes Table. Table recording planned secondary outcomes as reported in retrieved studies.

\section{Acknowledgements \\ Dr. Bagshaw is supported by a Canada Research Chair in Critical Care Nephrology. Dr. Silver is supported by a Kidney Research Scientist Core Education and National Training (KRESCENT) Program New Investigator Award (co-funded by the Kidney Foundation of Canada, Canadian Society of Nephrology, and Canadian Institutes of Health Research).}

\section{Authors' contributions}

All authors contributed to study conception and design. RF and MS conducted the literature review. RJK and AA collected and extracted the data. BVM performed the analysis. All authors contributed to drafting the paper and approving the final manuscript.

\section{Funding}

This work was supported by the Alberta Strategy for Patient-Oriented Research (SPOR) SUPPORT Unit Knowledge Translation Platform, which is funded by the Alberta Innovates and the Canadian Institutes of Health Research. This work was supported by the University of Alberta Foundation Kaye Fund. 


\section{Availability of data and materials}

All data generated or analyzed during this study are included in this published article.

\section{Ethics approval and consent to participate}

This study is a systematic review and meta-analysis, and ethics approval and consent to participate are not available.

\section{Consent for publication}

Not available.

\section{Competing interests}

Dr. Bagshaw reports having received speaking fees and unrestricted funding support from Baxter Healthcare Corp.

Dr. Gibney reports having received speaking fees from Baxter Healthcare. Dr. Silver reports having received speaking fees from Baxter Healthcare.

\section{Author details}

'Department of Critical Care Medicine, Faculty of Medicine and Dentistry, University of Alberta, 2-124E Clinical Sciences Building 8440112 St. NW, Edmonton, Alberta T6G 2B7, Canada. ${ }^{2}$ Alberta Strategy for Patient-Oriented Research (SPOR) SUPPORT Unit Knowledge Translation Platform, University of Alberta, 4-472 Edmonton Clinic Health Academy, 11405 - 87 Avenue, Edmonton, Alberta T6G 1C9, Canada. ${ }^{3}$ Alberta Research Center for Health Evidence (ARCHE), University of Alberta, 4-496 Edmonton Clinic Health Academy, 11405 - 87 Avenue, Edmonton, Alberta T6G 1C9, Canada. ${ }^{4}$ Division of Nephrology, Department of Medicine, Queen's University, 94 Stuart Street, Kingston, Ontario K7L 3N6, Canada.

\section{Received: 20 August 2019 Accepted: 27 January 2020}

\section{Published online: 13 February 2020}

\section{References}

1. Hoste EA, Bagshaw SM, Bellomo R, Cely CM, Colman R, Cruz DN, Edipidis K, Forni LG, Gomersall CD, Govil D, et al. Epidemiology of acute kidney injury in critically ill patients: the multinational AKI-EPI study. Intensive Care Med. 2015;41(8):1411-23.

2. Prowle JR, Bellomo R. Continuous renal replacement therapy: recent advances and future research. Nat Rev Nephrol. 2010;6(9):521-9.

3. Hsu RK, McCulloch CE, Dudley RA, Lo LJ, Hsu CY. Temporal changes in incidence of dialysis-requiring AKI. J Am Soc Nephrol. 2013;24(1):37-42.

4. Nct: Standard vs. Accelerated Initiation of RRT in Acute Kidney Injury (STARRT-AKl: principal Trial). https://clinicaltrials.gov/show/nct02568722. 2015.

5. Gaudry S, Hajage D, Schortgen F, Martin-Lefevre L, Pons B, Boulet E, Boyer A, Chevrel G, Lerolle N, Carpentier D, et al. Initiation strategies for renalreplacement therapy in the intensive care unit. $\mathrm{N}$ Engl J Med. 2016;375(2):122-33.

6. Zarbock A, Kellum JA, Schmidt C, Van Aken H, Wempe C, Pavenstadt H, Boanta A, Gerss J, Meersch M. Effect of early vs delayed initiation of renal replacement therapy on mortality in critically ill patients with acute kidney injury: the ELAIN randomized clinical trial. JAMA. 2016;315(20):2190-9.

7. Ferreira JA, Johnson DW. The incidence of thrombocytopenia associated with continuous renal replacement therapy in critically ill patients. Ren Fail. 2015;37(7):1232-6.

8. Guru PK, Singh TD, Akhoundi A, Kashani KB. Association of thrombocytopenia and mortality in critically ill patients on continuous renal replacement therapy. Nephron. 2016;133(3):175-82

9. Hoste EA, Blot SI, Lameire NH, Vanholder RC, De Bacquer D, Colardyn FA. Effect of nosocomial bloodstream infection on the outcome of critically ill patients with acute renal failure treated with renal replacement therapy. J Am Soc Nephrol. 2004;15(2):454-62.

10. Ronco C, Bellomo R. Complications with continuous renal replacement therapy. Am J Kidney Dis. 1996;28(5):S100-4.

11. Silversides JA, Pinto R, Kuint R, Wald R, Hladunewich MA, Lapinsky SE, Adhikari NK. Fluid balance, intradialytic hypotension, and outcomes in critically ill patients undergoing renal replacement therapy: a cohort study. Crit Care. 2014;18(6):624.

12. van de Wetering J, Westendorp RG, van der Hoeven JG, Stolk B, Feuth JD, Chang PC. Heparin use in continuous renal replacement procedures: the struggle between filter coagulation and patient hemorrhage. J Am Soc Nephrol. 1996;7(1):145-50.

13. Cerdá J, Liu KD, Cruz DN, Jaber BL, Koyner JL, Heung M, Okusa MD, Faubel S. Promoting kidney function recovery in patients with AKI requiring RRT. Clin J Am Soc Nephrol. 2015;10(10):1859-67.

14. Schiffl H. Renal recovery after severe acute renal injury. Eur J Med Res. 2008; 13(12):552-6.

15. Ferreira V, Neto MM, da Cardeal Costa JA. Association of Infections with the use of a temporary double-lumen catheter for hemodialysis. Nephrol Nurs J. 2018;45(3):261-7.

16. Khwaja A. KDIGO clinical practice guidelines for acute kidney injury. Nephron Clin Pract. 2012;120(4):c179-84.

17. Yoshida T, Matsuura R, Komaru Y, Miyamoto Y, Yoshimoto K, Hamasaki Y, Noiri E, Morimura N, Nangaku M, Doi K. Kinetic estimated glomerular filtration rate as a predictor of successful continuous renal replacement therapy discontinuation. Nephrol. 2019;24(3):287-93.

18. Han SS, Bae E, Song SH, Kim DK, Kim YS, Han JS, Joo KW. NT-proBNP is predictive of the weaning from continuous renal replacement therapy. Tohoku J Exp Med. 2016;239(1):1-8.

19. Chen X, Chen Z, Wei T, Li P, Zhang L, Fu P. The effect of serum neutrophil gelatinase-associated lipocalin on the discontinuation of continuous renal replacement therapy in critically ill patients with acute kidney injury. Blood Purif. 2019;48(1):10-17.

20. Frohlich S, Donnelly A, Solymos O, Conlon N. Use of 2-hour creatinine clearance to guide cessation of continuous renal replacement therapy. J Crit Care. 2012;27(6):744.e741-5.

21. Uchino S, Bellomo R, Morimatsu H, Morgera S, Schetz M, Tan I, Bouman C, Macedo E, Gibney N, Tolwani A, et al. Discontinuation of continuous renal replacement therapy: a post hoc analysis of a prospective multicenter observational study. Crit Care Med. 2009;37(9):2576-82.

22. Katayama S, Uchino S, Uji M, Ohnuma T, Namba Y, Kawarazaki H, Toki N, Takeda K, Yasuda H, Izawa J, et al. Factors predicting successful discontinuation of continuous renal replacement therapy. Anaesth Intensive Care. 2016:44(4):453-7.

23. Gleeson PJ, Crippa IA, Sexton DJ, Fontana V, Taccone F, Creteur J, Vincent JL. Determinants of renal recovery and mortality in patients undergoing continuous renal replacement therapy in the ICU. Intensive Care Med Exp. 2015;3:A54.

24. Dewitte A, Joannes-Boyau O, Sidobre C, Fleureau C, Bats ML, Derache P, Leuillet S, Ripoche J, Combe C, Ouattara A. Kinetic eGFR and novel AKI biomarkers to predict renal recovery. Clin J Am Soc Nephrol. 2015;10(11): 1900-10.

25. Aniort J, Ait Hssain A, Pereira B, Coupez E, Pioche PA, Leroy C, Heng AE, Souweine $B$, Lautrette A. Daily urinary urea excretion to guide intermittent hemodialysis weaning in critically ill patients. Crit Care. 2016;20:43.

26. Viallet N, Brunot V, Kuster N, Daubin D, Besnard N, Platon L, Buzancais A, Larcher $\mathrm{R}$, Jonquet $\mathrm{O}$, Klouche $\mathrm{K}$. Daily urinary creatinine predicts the weaning of renal replacement therapy in ICU acute kidney injury patients. Ann Intensive Care. 2016;6(1):71.

27. Raurich JM, Llompart-Pou JA, Novo MA, Talavera C, Ferreruela M, Ayestaran I. Successful weaning from continuous renal replacement therapy. Associated risk factors. J Crit Care. 2018;45:144-8.

28. Kim CS, Bae EH, Ma SK, Kim SW. A prospective observational study on the predictive value of serum cystatin C for successful weaning from continuous renal replacement therapy. Kidney Blood Press Res. 2018:43(3):872-81.

29. Yang T, Sun S, Lin L, Han M, Liu Q, Zeng X, Zhao Y, Li Y, Su B, Huang S, et al. Predictive factors upon discontinuation of renal replacement therapy for long-term chronic dialysis and death in acute kidney injury patients. Artif Organs. 2017;23:23.

30. Yang T, Sun S, Zhao Y, Liu Q, Han M, Lin L, Su B, Huang S, Yang L. Biomarkers upon discontinuation of renal replacement therapy predict 60 day survival and renal recovery in critically ill patients with acute kidney injury. Hemodial. 2017;11:11

31. Zhang $Z, X u X, N i$, Jin N. Serum cystatin $C$ is associated with renal function recovery in critically ill patients undergoing continuous renal replacement therapy. Nephron. 2012;122(3-4):86-92.

32. Higgins JPT TJ, Chandler J, Cumpston M, Li T, Page MJ, Welch VA: Cochrane Handbook for Systematic Reviews of Interventions. In. Edited by JPT H. Cochrane; 2019.

33. Moher D, Liberati A, Tetzlaff J, Altman DG. Preferred reporting items for systematic reviews and meta-analyses: the PRISMA statement. PLoS Med. 2009;6(7):e1000097. 
34. Al Saadon A, Katulka R, Sebastianski M, Featherstone R, Vandermeer B, Gibney RTN, Rewa OG, Bagshaw SM. Determining the optimal time for liberation from renal replacement therapy in critically ill patients: protocol for a systematic review and meta-analysis (DOnE RRT). BM Open. 2018;8(11):e023306.

35. The Newcastle-Ottawa Scale (NOS) for assessing the quality of nonrandomised studies in meta-analyses .http://www.ohri.ca/programs/ clinical_epidemiology/oxford.asp. Accessed 19 Oct 2018.

36. Reitsma JB, Glas AS, Rutjes AW, Scholten RJ, Bossuyt PM, Zwinderman AH. Bivariate analysis of sensitivity and specificity produces informative summary measures in diagnostic reviews. J Clin Epidemiol. 2005;58(10):982-90.

37. Jeon J, Kim DH, Baeg SI, Lee EJ, Chung CR, Jeon K, Lee JE, Huh W, Suh GY, Kim YG, et al. Association between diuretics and successful discontinuation of continuous renal replacement therapy in critically ill patients with acute kidney injury. Crit Care. 2018;22(1):255.

38. Itenov TS, Berthelsen RE, Jensen JU, Gerds TA, Pedersen LM, Strange D, Thormar K, Loken J, Andersen MH, Tousi $\mathrm{H}$, et al. Predicting recovery from acute kidney injury in critically ill patients: development and validation of a prediction model. Crit Care Resusc. 2018;20(1):54-60.

39. Romero-Gonzalez G, Clark WR, Ferrari F, Lorenzin A, Ronco C. Factors related with effective discontinuation of CRRT in ICU. Blood Purif. 2017; 44(3):170-1.

40. Kim C, Oh T, Kim H, Yong U, Bae E, Ma S, Lee J, Kim S. Predictive value of cystatin C-based eGFR for successful weaning from continuous renal replacement therapy: a prospective observational study. In: Kidney Week 2016. vol. 27. Chicago: Journal of the American Society of Nephrology; 2016. p. $249 \mathrm{~A}$

41. Ohnuma T, Suzuki J, Sanayama H, Ito K, Fujiwara T, Yamada H, Sanui M. Higher urine output in patients with acute kidney injury after discontinuation of continuous renal replacement therapy is associated with lower mortality and improved renal recovery. Intensive Care Med. 2013;39:S442.

42. Heise D, Gries D, Moerer O, Bleckmann A, Quintel M. Predicting restoration of kidney function during CRRT-free intervals. J Cardiothorac Surg. 2012;7:6.

43. Solymos O, Frohlich S, Conlon N. Use of 2-hourly creatinine clearance to inform cessation of renal replacement therapy. Crit Care. 2011;15:S47.

44. Franzen D, Rupprecht C, Hauri D, Bleisch JA, Staubli M, Puhan MA. Predicting outcomes in critically ill patients with acute kidney injury undergoing intermittent hemodialysis--a retrospective cohort analysis. Int J Artif Organs. 2010;33(1):15-21.

45. Wu VC, Ko WJ, Chang HW, Chen YW, Lin YF, Shiao CC, Chen YM, Chen YS, Tsai PR, Hu FC, et al. Risk factors of early redialysis after weaning from postoperative acute renal replacement therapy. Intensive Care Med. 2008;34(1):101-8.

46. van der Voort PH, Boerma EC, Koopmans M, Zandberg M, de Ruiter J, Gerritsen RT, Egbers PH, Kingma WP, Kuiper MA. Furosemide does not improve renal recovery after hemofiltration for acute renal failure in critically ill patients: a double blind randomized controlled trial. Crit Care Med. 2009;37(2):533-8.

47. Ouellette DR, Patel S, Girard TD, Morris PE, Schmidt GA, Truwit JD, Alhazzani W, Burns SM, Epstein SK, Esteban A, et al. Liberation From Mechanical Ventilation in Critically III Adults: An\&\#xa0;Official American College of Chest Physicians/American Thoracic Society Clinical Practice Guideline: Inspiratory Pressure Augmentation During Spontaneous Breathing Trials,\&\#xa0; Protocols Minimizing Sedation, and Noninvasive Ventilation Immediately After Extubation. Chest. 2017;151(1):166-80.

48. Boles J-M, Bion J, Connors A, Herridge M, Marsh B, Melot C, Pearl R, Silverman $\mathrm{H}$, Stanchina M, Vieillard-Baron A, et al. Weaning from mechanica ventilation. Eur Respir J. 2007;29(5):1033-56.

49. Citerio G, Bakker J, Bassetti M, Benoit D, Cecconi M, Curtis JR, Hernandez G, Herridge $M$, Jaber $S$, Joannidis $M$, et al. Year in review in Intensive Care Medicine 2013: I. Acute kidney injury, ultrasound, hemodynamics, cardiac arrest, transfusion, neurocritical care, and nutrition. Intensive Care Med. 2014;40(2):147-59.

50. Fealy N, Aitken L, Toit E, Baldwin I. Continuous renal replacement therapy: current practice in Australian and New Zealand intensive care units. Crit Care Resusc. 2015;17(2):83-91.

51. Rewa OG, Villeneuve PM, Lachance P, Eurich DT, Stelfox HT, Gibney RTN, Hartling L, Featherstone R, Bagshaw SM. Quality indicators of continuous renal replacement therapy (CRRT) care in critically ill patients: a systematic review. Intensive Care Med. 2017:43(6):750-63.
52. Schiffl $\mathrm{H}$. Discontinuation of renal replacement therapy in critically ill patients with severe acute kidney injury: predictive factors of renal function recovery. Int Urol Nephrol. 2018;50(10):1845-51.

53. Klouche K, Gibney RTN, Forni LG. Can this patient be safely weaned from RRT? Intensive Care Med. 2018;44(5):639-42.

\section{Publisher's Note}

Springer Nature remains neutral with regard to jurisdictional claims in published maps and institutional affiliations.
Ready to submit your research? Choose BMC and benefit from:

- fast, convenient online submission

- thorough peer review by experienced researchers in your field

- rapid publication on acceptance

- support for research data, including large and complex data types

- gold Open Access which fosters wider collaboration and increased citations

- maximum visibility for your research: over $100 \mathrm{M}$ website views per year

At BMC, research is always in progress.

Learn more biomedcentral.com/submissions 